\title{
HANDWRITING ANALYZER AND ANALYSES OF HUMAN HANDWRITING MOVEMENTS
}

\author{
MAKOTO YASUHARA
}

The University of Electro-Communications

\begin{abstract}
A handwriting analyzer is presented, which generates $\mathrm{X}-\mathrm{Y}$ coordinate together with a writing pressure of a pencil point during handwriting movement. The combination of these two functions using computer system might be quite effective especially for psychological and medical diagnoses or experiments. And further, a dynamic model of handwriting process is proposed, which plays an important role in the active analysis of human handwriting process. The values of parameters in the model are guessed experimentally.
\end{abstract}

\section{INTRODUCTION}

A computational program for handwriting analysis and synthesis called "active analysis" is proposed. This active analysis would enable us to introduce a new method to automatic or mechanical graphoanalysis. The justice for the application of the idea of active recognition (Liberman, 1959, 1962; Stevens, 1960) widely acknowledged by speech researchers to handwriting research has been discussed from various view points (Lindgren, 1965; Yasuhara, 1966). The system for the mechanical graphoanalysis is considered to be associated with the active facilities that allow itself to generate handwritings if necessary. These ideas led the author to the proposal of the dynamic model of handwriting process. The handwriting analyzer is designed for the input device to this handwriting simulator as well as for the analyses of actual human handwriting movements. In the present paper, the details of the handwriting analyzer are described and then the optimal values of the parameters that appear in the proposed dynamic model of handwriting process are guessed experimentally.

1 The author wishes to express here his deep gratitude to prof. Hiroshi Sato for valuable suggestions.

\section{Handwriting ANALyzer}

First of all, we must measure the displacement, velocity, acceleration of handwriting movements and the writing pressure of the pencil point using a device for transforming $\mathrm{X}, \mathrm{Y}$ coordinates and a pressure of a pencil point into electrical signals as functions of time. Handwriting analyzer generates such signals and these signals can be connected to $\mathrm{X}, \mathrm{Y}$ and $\mathrm{Z}$ axes of the cathode ray tube display as well as to an input channel of the computer.

The principle for picking up these informations of the stylus is illustrated in Fig. 1. A writing plate consists of a square of uniform resistive material fed with equal magnitude current. The contacts are identically shaped and equally spaced along the four edges. An uniform

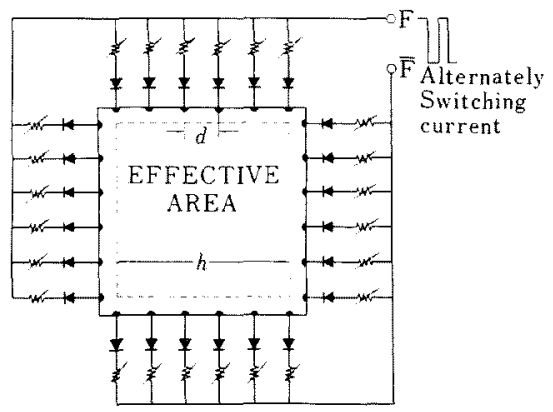

Fra. 1. The principle of an $\mathrm{X}-\mathrm{Y}$ position transducer. 
horizontal and vertical current flow are established alternately by switching the operational mode of the respective contact sets. For the contact separation $\mathrm{d}$ is small compared with the writing plate width $h$, there will be an uniform potential gradient between mutually faced contact sets except for local deformations in the vicinity of edges. Thus, the time variant analogue samples of $\mathrm{X}, \mathrm{Y}$ coordinates are generated by placing or moving the stylus over the resistive surface. A durable, highly transparent stannic oxide $\left(\mathrm{SnO}_{2}\right)$ coating on a carefully polished plate of glass as a base shows no physical or chemical deterioration after several months of use. The stylus used has not so hard a pencil lead and it can move freely over the coating. The writing plate is sustained at the four corners by the pressure sensitive gaugesthe strain gauges of non-contact type are

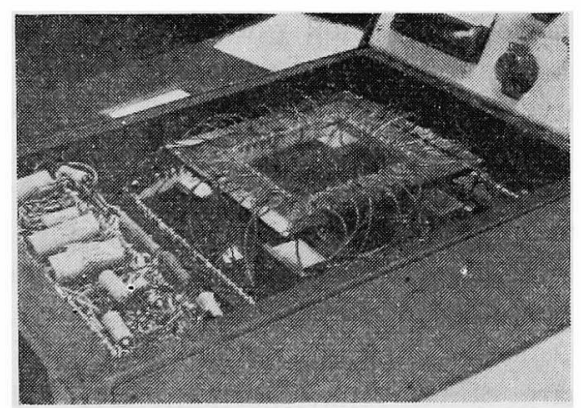

FIG. 2. The photograph of the two dimensional resistive surface.

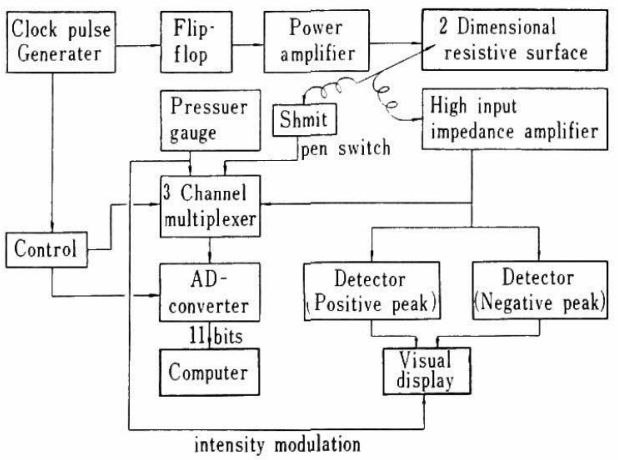

FIG. 3. The system block diagram of handwriting analyzer. used-and the signals from them are summed each other.

The multiplexer and analogue to digital converter convert simultaneously 3 channels of analogue signals corresponding to $\mathrm{X}, \mathrm{Y}$ coordinates of the stylus and the writing pressure $Z$ respectively into 11 bits digital samples at a sampling rate of 400 $\mathrm{Hz}$.

Fig. 2 shows the photograph of two dimensional resistive surface and Fig. 3 is the system block diagram. Fig. 4 shows the electrical potential distribution generated over the resistive surface, showing rather linear potential gradient in the central portion. Fig. 5 shows one of the

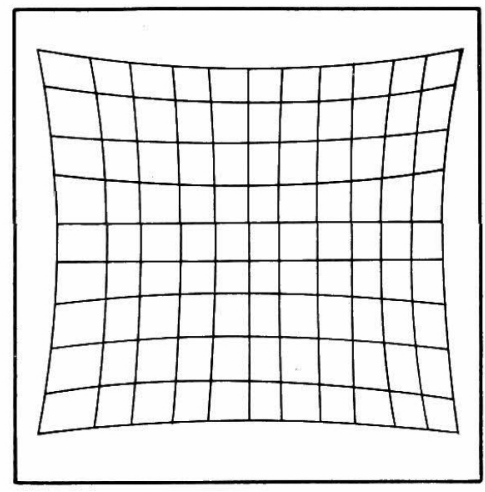

FIG. 4. The electrical potential distribution generated over the resistive surface $(120 \mathrm{~mm} \times$ $120 \mathrm{~mm}$ ). The central part is selected to use.

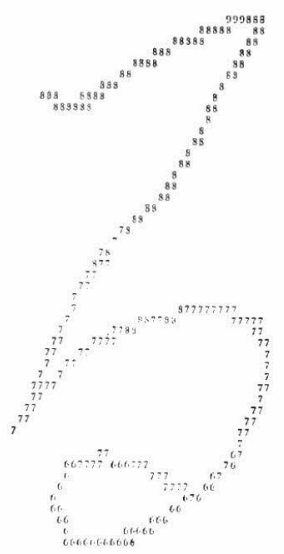

FIG. 5. Japanese letter る uttered " ru " which is transferred to the computer, representing the writing pressure by the printed numerics. 


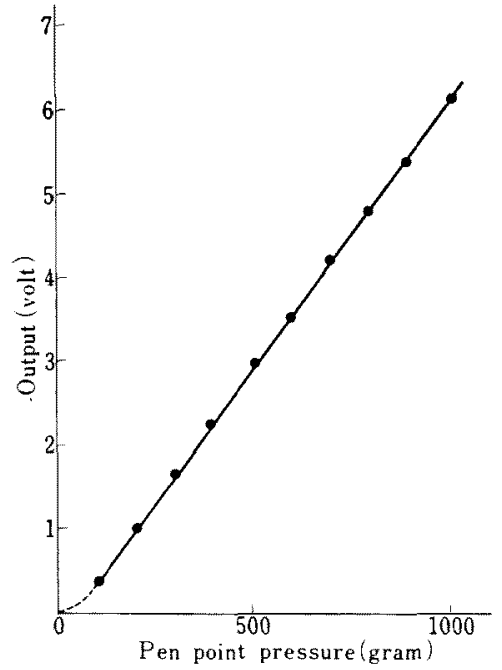

Fig. 6. The output characteristics of the pressure gauge.
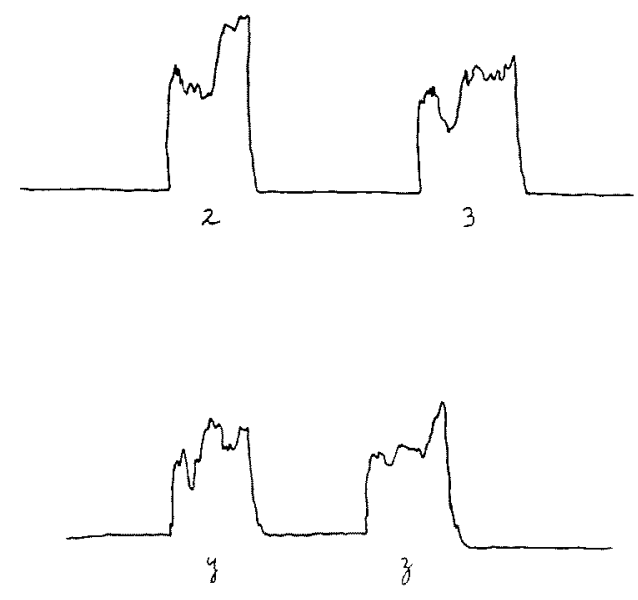

1 sec.

FIG. 7. Some examples of the measured waveforms of the writing pressure.

examples that is transferred to the computer, intending to represent the Japanese letter る uttered "ru". The pen point pressure is also shown in the figure by the printed numerics. Fig. 6 illustrates the output characteristics of the pressure gauge, showing almost complete linearity from $100 \mathrm{~g}$ to $1000 \mathrm{~g}$. In most cases, this range covers the actual human handwriting pressure. Fig. 7 represents some examples of the measured writing pressure waveforms.

\section{The Dynamic Model of Handwriting Process}

Active analysis of handwriting signals will be performed by comparison of the given signal with the signal generated by the generating program of handwriting simulated model of handwriting process. In the course of above comparing process, the parameters of handwriting signals such as timings of the occurrence of motor commands of writing movements would be extracted. These parameters would serve as the important distinctive features of the individual handwriting.

Cursive handwriting can be considered as a highly skilled process which is executed by means of a rapid sequence of motion. Such processes were studied as early as 1917 by Lashley. An important principle of the process is the principle of position feedback. No doubt this principle is used in guiding human movements to a certain extent, but it is well-known that it does not always hold for quick and well-practiced movements. Lashley concluded that an effector mechanism can be pre-set to discharge at a given intensity or for a given duration, independent of any sensory control. According to his opinions, this holds also for the initiation and timings of contraction of the different muscles in complex movements. He postulated some central nervous mechanisms which activated different muscles with predetermined intensity and duration in predetermined order. Thus, the proposal of the following two assumptions would be justified;

1) The effects of position feedback can be neglected for fast cursive handwriting,

2) The timings of muscle contraction control the topological shape of a pattern, independent of the magnitude of the applied forces.

Since the model needs to contain no feedback route by these assumptions, the 


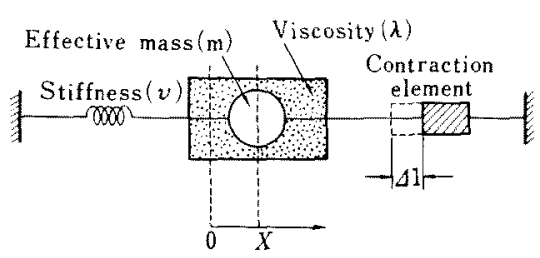

Fro. 8. Schematic illustration of the handwriting model.

motion activated between two neighbouring control signals from the central nervous system must be arranged only by the transmission characteristics of the hand itself.

Hand operating system, containing the muscle as an effector, can be described as the dynamic process expressed by the mechanical, electrical or mathematical model. We propose a mathematical model of the dynamics of handwriting. Fig. 8 schematizes the proposed model of handwriting process in which the handpen couple is considered as a mass point with a equivalent mass $m$ that moves according to the dynamic equations (1), where the handwriting patterns are assumed to be the resultant locus of the movement of the equivalent mass point.

$$
\left.\begin{array}{l}
\ddot{X}+r_{x} \dot{X}+k_{x} X=F_{x}(t), \\
\ddot{Y}+r_{y} \dot{Y}+k_{y} Y=F_{y}(t),
\end{array}\right\}
$$

where the effective viscosties $r_{x}, r_{y}$ and the effective stiffnesses of muscle $k_{x}, k_{y}$ are given as follows;

$$
\begin{aligned}
& r_{x}=\lambda_{x} / m+\mu_{x}\{1+P(t)\} / v, \\
& r_{y}=\lambda_{y} / m+\mu_{y}\{1+P(t)\} / v \text {, } \\
& k_{x}=v_{x} \mid m \text {, } \\
& k_{y}=v_{y} / m \\
& v=\left(\dot{X}^{2}+\dot{Y}^{2}\right)^{1 / 2} \text { writing speed, } \\
& \lambda \text { viscosity, } \\
& \mu \quad \text { dynamic friction } \\
& \text { - coefficient of } \\
& \text { stiffness. }
\end{aligned}
$$

Assuming the pen point pressure $P(t)$ and the writing speed $v$ to be time-invariant, the effective viscosities $r_{x}, r_{y}$ are proved to be time invariant.

Here let us introduce further simplification by neglecting the stiffness term which is actually considered to be of less importance compared with the viscosity term. So the simplified model will be expressed by the following equations;

$$
\left.\begin{array}{rl}
\ddot{X}+r_{x} \dot{X} & =F_{x}(t), \\
\dot{Y}+r_{y} \dot{Y} & =F_{y}(t) .
\end{array}\right\}
$$

As noted previously, the paired force functions $F_{x}(t), F_{y}(t)$ are the most important information bearing elements. Especially the timings of transitions of these functions exactly determine the topological features of the pattern to be generated, while their magnitudes are only related to a proportional change in the size of the pattern.

\section{The Optimal Estimation of The Model Parameters}

Now we must determine the values of $r_{x}, r_{y}$ in eqs. (2) which specify the model behavior. Thus we executed the following experiment.

Subjects were instructed to make their resting pencil point run to and fro on the table at $t=0$ with their preferred writing speed and pressure. The time variant coordinate samples generated by the handwriting analyzer were transferred to the computer at the sampling rate of $400 \mathrm{~Hz}$. If the muscular force is considered to build up exponentially at $t=0$, the following equations must be hold;

$$
\left.\begin{array}{l}
\ddot{X}+r \dot{X}=A\left(1-e^{-t / \tau}\right), \\
\dot{X}(0)=X(0)=0 .
\end{array}\right\}
$$

accordingly,

$$
\begin{aligned}
X(t, r, \tau, A)= & A\left[-(1+r \tau) / r^{2}+t / r\right. \\
& -\tau^{2} e^{-t / \tau} /(1-r \tau) \\
& \left.+e^{-r t} / r^{2}(1-r \tau)\right] .
\end{aligned}
$$




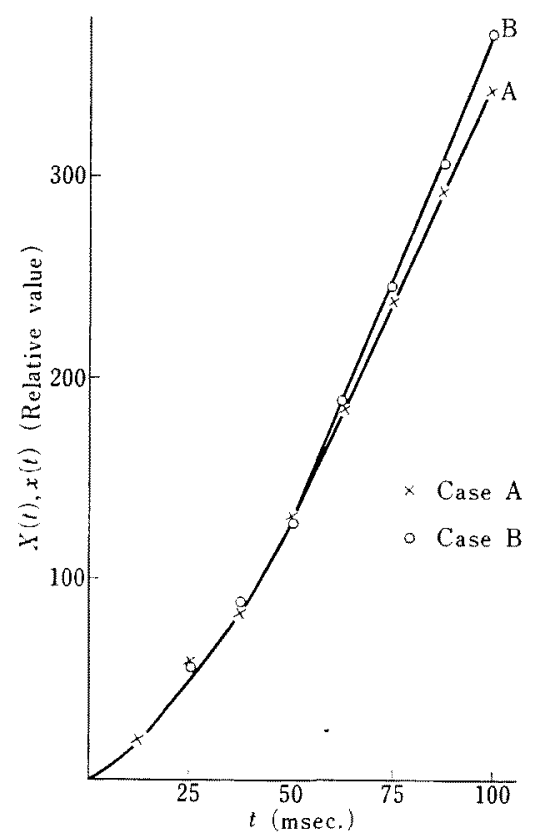

FIG. 9. The goodness of fitting $X(t, r, \tau, A)$ to $x(t)$. Subject Male, a 22 years old student.
Let $x(t)$ represent the experimentally obtained coordinate function, and further let us introduce the integral functional $J$ which evaluates the distance between the experimental and the theoretical function;

$$
\begin{aligned}
J(r, \tau, A)= & 1 / w \int_{0}^{W}\{X(t, r, \tau, A) \\
& -x(t)\}^{2} d t .
\end{aligned}
$$

Our concern here is to find the optimal values of $r, \tau$ and $\mathrm{A}$ which minimize the functional $J$. Fig. 9 illustrates the goodness of fitting $X(t, r, \tau, A)$ to $x(t)$ for two cases A, B of a subject (male, a 22 years old student), in which the extracted optimal values of the parameter $r, \tau$ and $A$ calculated by the iteration method were $r^{*}=18.6 \mathrm{~Hz}, \tau^{*}=.93 \mathrm{msec}, A^{*}=2298$ for case $\mathrm{A}$ and $r^{*}=19.2 \mathrm{~Hz}, \tau^{*}=.94 \mathrm{msec}$, $A^{*}=2305$ for case B.

Fig. 10 illustrates the initial one cycle of to-and-fro movement of the hand in which the first and the second derivatives of

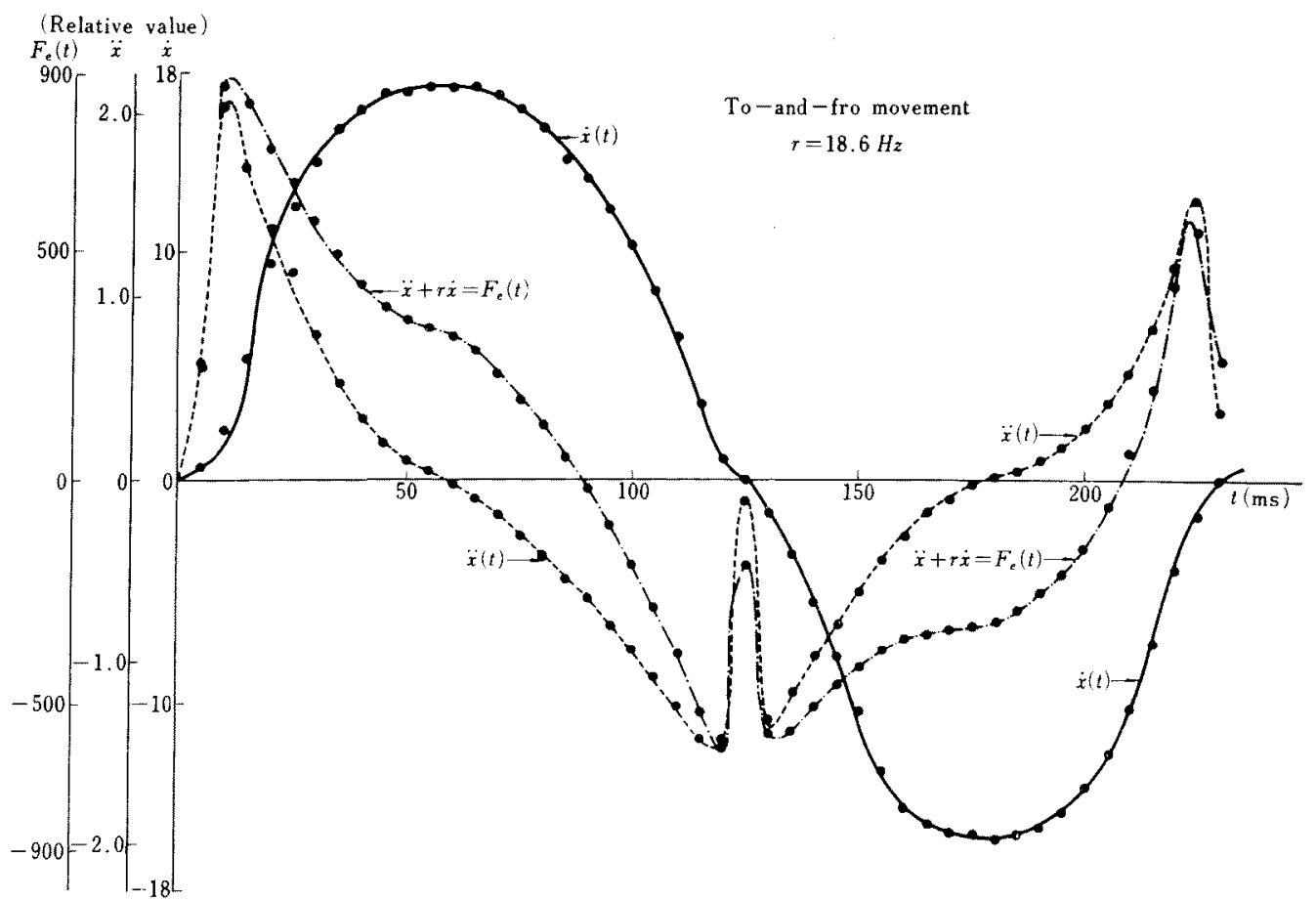

FIG. 10. To-and-fro movement of the hand. 
(A)

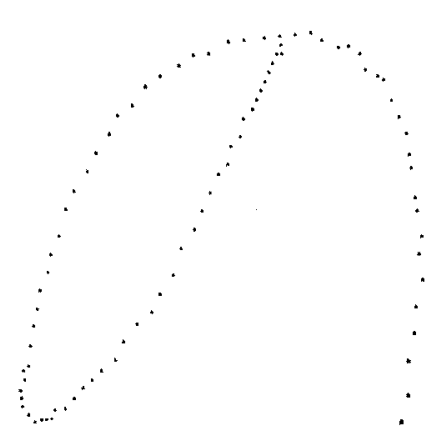

(B)
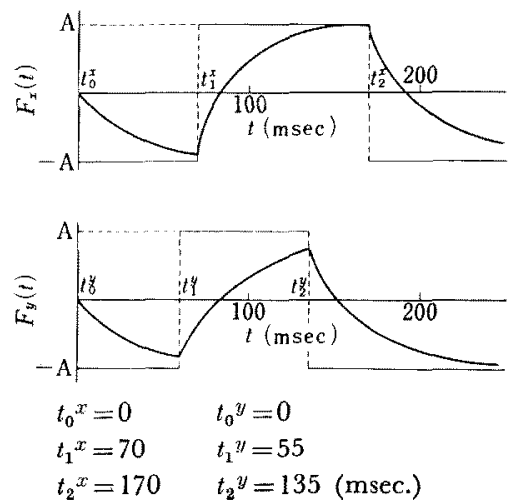

Fic. 11. The synthesized Japanese letter 0 uttered " no" (A) and its applied force functions (B).

displacement and the estimated force function $F_{e}(t)=\ddot{X}+18.6 \dot{X}$ for case $A$ are plotted. First of all, the dips in $\ddot{X}(t)$ at $\dot{X}(0)=0$ (except at $t=0$ ) directly suggest that the static friction force between the pencil and the table is rather effective, which shows the significance of writing pressure in human handwriting process. The initial behavior of the force function is quite different from those of the other parts because the static friction effect is more prominent, that is, the initial steep and rather high peak of $F_{e}(t)$ shows fluently the fact that the initial force must be greater than the static friction force and therefore build up so quickly. The above-calculated optimal value $\tau^{*}$ is considered to correspond to this building up time. The transition time from one level to another can be estimated about $40 \mathrm{msec}$ according to this data. This is quite a reasonable value compared with the other data obtained by physiological measurements (Bourne, 1960).

In conclusion, The presented handwriting analyzer generates $\mathrm{X}, \mathrm{Y}$ coordinates together with a writing pressure of a pencil point. Both techniques are well known, but only separately. The combination of these two functions using computer systems would be quite effective especially for psychological or medical diagnoses and experiments.
The application of the active method to the recognition of a handwriting pattern or to the graphological analyses as well as speech analyses would surely be fruitful. It must be indispensable especially for mechanical graphoanalysis to catch the informations about the actual handwriting movements. The proposed dynamic model of handwriting process would be powerful as a guiding of our future analyzer-synthesizer system of handwriting recognition or graphological analysis of handwriting, which is based on Stevens's analysis-by-synthesis technique.

The author presents lastly one of the examples of the synthesized Japanese letter by the established handwriting model in Fig. 11-A. Fig. 11-B illustrated the applied force functions $F_{x}(t), F_{y}(t)$, showing the timings of the occurrence of the control commands by $t_{i}^{x}, t_{j}^{y}(i, j=0$, $1,2, \ldots)$. We obtained $X(t)$ and $Y(t)$ from the eqs. (2), where $r_{x}=r_{y}=18.6 \mathrm{~Hz}$ was chosen.

Thus we could synthesize almost any handwriting patterns by giving suitable values of $t_{i}^{x}$ and $t_{j}^{y}$.

\section{REFERENCES}

Bourne, G. H. 1960 The structure and functions of muscle vol. I, II Academic press.

LASHLEY, K.S. 1917 The accuracy of movements in the absense of excitation from the moving 
organ. Amer. 7. Physiol. 43, 169-194.

Liberman, A. M. 1959 Some results of research on speech perception. J. accoust. Soc. Amer., 31, 1490-1499.

Liberman, A. M. 1962 A motor theory of speech perception, Speech communication seminar, Stockholm.

LINDGREN, N. 1965 Machine recognition of human language, part III, IEEE Spectrum.

Stevens, K. N. 1960 Toward a model for speech recognition. J. accoust. Soc. Amer., 32, 47-51.

YasuHARA, M. 1966 Notes on the active recognition and the model of pattern recognition Annual report. Res. Lab. of Comm. Sci.

(Received June 30, 1969) 